\title{
Robust collaborative collision avoidance between robots with nearly symmetric crossing trajectories
}

\author{
Grimaldo Silva ${ }^{3}$, Khansa Rekik ${ }^{2}$, James Crowley ${ }^{1}$
}

\begin{abstract}
The growth in both acceptance and usage of mobile robots have given risen to novel challenges in robot navigation. Often, robots that share a space but are unable to communicate are required to safely avoid each other even under sensor noise. Current approaches have often relied on the assumption that collaboration is always done correctly, in practice, sensor noise might lead robots to make avoidance motions that are not mutually beneficial and do not actually decrease the collision risk. Our approach intends to mitigate the negative impact of sensor noise in collaborative collision avoidance of robots. As a consequence, even if robots initially take non-mutually beneficial avoidance motions they would correctly perceive their role in the next decision step.
\end{abstract}

\section{INTRODUCTION}

Mobile robots have seen an increased usage in recent years and this trend should continue to hold for the foreseeable future. Considering the multitude of models and specifications, this work focuses on robots that share a space but are unable to directly communicate their intentions during collision avoidance motions. In other words, robots should communicate solely through motion and still be able to avoid collision with each other in a safe and preferably collaborative manner. Collaboration is important for two reasons. First it provides a solution for the classic freezing robot problem [1] and second that sharing motion adaptations among agents in the environment leads to overall smaller and more readable motion adaptations from individual agents.

Effective collaboration requires that any pairwise combination of robots involved in a collision avoidance situation agree on the side in which they will cross each other in order to avoid collision. Choosing a specific side to cross indicates which robot crosses in front and which crosses behind the other i.e. their roles. However, without direct communication a convention has to be established in order to allow for same crossing side to be reliably chosen by both robots.

The sign of the derivative of the bearing angle has been used to reliably predict crossing side [2], [3] in the literature. However, as robots that are unable to directly or indirectly communicate are subject to noise in their perception of this derivative, the sign of this shared value may be perceived differently by each robot which leads them to incorrectly attempt to cross each other on different sides - what we call an ineffective collaboration.

\footnotetext{
${ }^{1}$ Univ. Grenoble Alpes, Inria, CNRS, Grenoble INP, LIG, 38000 Grenoble, France

${ }^{2}$ ZeMA gGmbH, Gewerbepark, Eschbergerweg 46, 66121 Saarbrücken

${ }^{3}$ Lactec, Parque Tecnológico da Bahia, 41745715, SSA, Brasil

jose.jgrimaldo@gmail.com, K.rekik@zema.de, James.Crowley@inria.fr 978-1-6654-1213-1/21/\$31.00 @2021 IEEE
}

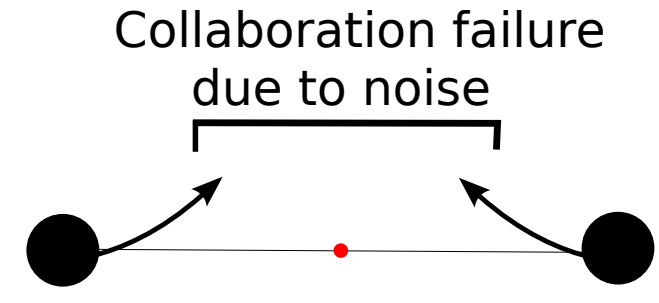

Our approach

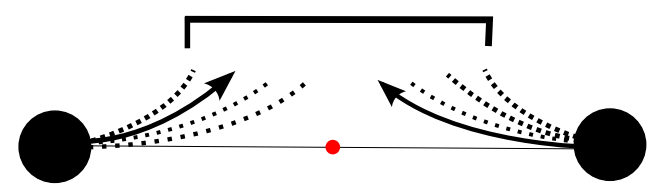

Fig. 1: Noise in the observation of the obstacles position and motion can lead to incorrect collaborative motion. Our approach reduces both duration and impact of this failure.

This ineffective collaboration is more prominent in socalled near symmetry scenarios. Situations in which the derivative of the bearing angle is near zero and thus robots are unable to assess their crossing side with the required confidence. As shown in Fig. 1, our approach mitigates the chance of ineffective collaboration in these scenarios while still attempting to avoid collision. For that end, our approach models the error in observation of the derivative of the bearing angle, which is then used to obtain a confidence in the sign of the derivative of the bearing angle. This confidence is then used as an input into a collision avoidance approach in order to mitigate the chance of ineffective collaboration in the next time step. Our approach is evaluated in ROS simulated robots in scenarios with two to three robots.

\section{LITERATURE REVIEW}

Although our method focuses on robot-robot interaction, this section provides an overview on collaborative approaches for collision avoidance for different types of agent. As such, methods that deal with people, robots or any combination thereof are described as long as they provide important landmarks into collaborative collision avoidance.

Classical approaches have often attempted to guarantee safe navigation in the presence of static and moving obstacles [4] where the latter obstacles do not react to the presence nor motion of the robot. Some recent methods, able to effectively navigate even with sensor noise and in crowded scenarios [5], have also often relied on this assumption, in contrast, our 
approach focuses on scenarios with an unbounded number of robots that take (if able) reactive steps to avoid collision while dealing with sensor noise.

Although several collaborative approaches have been proposed a common limitation is that, in the absence of additional obstacles, agents would always preserve their current role as seen in both velocity object methods [6], [7] and social force model methods [8], [9]. Approaches based on synthetic vision, such as [3], [10], that are able to replicate several important characteristics of human vision and its impact on their motion, also rely on the assumption that roles are unchanging under repeated initial conditions.

More recent approaches have attempted to plan collision avoidance behavior while accommodating different role assignments. For instance, the concept of joint trajectories encode the collaborative aspect of collision avoidance between people [11] and were able encode both current and reversed role trajectories. However, this implicitly relied on the assumption that roles are always agreed upon in any pairwise interaction before collision avoidance starts - an assumption that is relaxed in this work.

Accepting that roles are sometimes reversed reveals a subtle issue [12]: whenever in any pairwise interaction only a single agent reverses their role ineffective collaboration is produced. A recent approach to this issue [12] considered that only a person can mistake its role. In our work a general model is proposed based on a sensor-specific error model where any robot can be at fault.

\section{COLlaboration IN COLLISION AVOIDANCE}

Collaboration during collision avoidance requires a protocol that robots follow in order to take effective actions. In this section the manner in which a robot can infer its role during avoidance is presented alongside its potential shortcomings.

\section{A. Derivative of the bearing angle}

The bearing angle represents the angle between the current heading of a robot and another obstacle (moving or otherwise). Its derivative, in [2], was found to be a reliable indicator of the side agents would cross each other and in conjunction with the change in size of an object over time [13] it can also be used in practical applications to predict future collision [3]. The behavior of the derivative of the bearing angle in near collision situations is shown in Fig. 2.

\section{B. Homotopy class decision boundary}

Given any number of agents involved in a crossing scenario, the side in which each pairwise combination of agents pass each other is an homotopy class [11]. More precisely, the decision of agents $r$ and $i$ of passing each other on right or on the left side will influence the sign of the integral over time of their derivative of the bearing angle

Effective collision avoidance requires that agents attempt to pass each other on the same side, that is, make the same homotopy class decision. In experiments with people, the derivative of the bearing angle has been shown to reliably predict the homotopy class decision of an agent [12] before

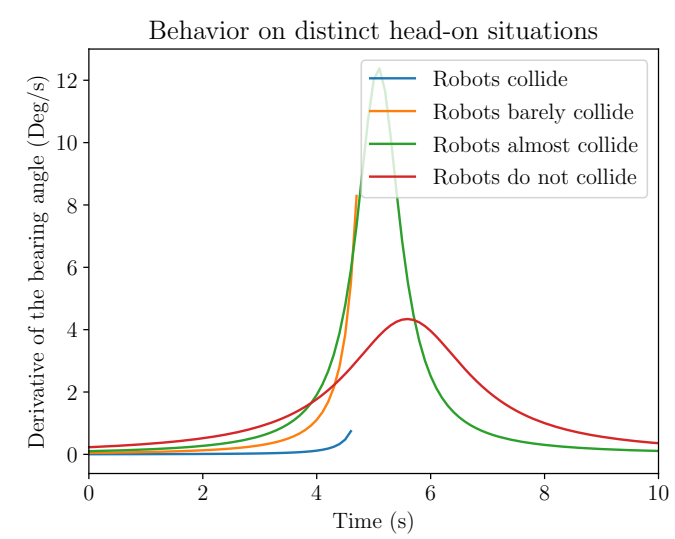

Fig. 2: The derivative of the bearing angle over time in four distinct head-on collision scenarios. Robots circumscribed radius is $0.8 \mathrm{~m}$ and move at $1.2 \mathrm{~m} / \mathrm{s}$. Closest distance between robots center points are, from highest to lowest risk of collision scenarios, exactly $0.1 \mathrm{~m}, 1.0 \mathrm{~m}, 2.2 \mathrm{~m}, 6.0 \mathrm{~m}$.

any collision avoidance action has been made. Based on this, a simplified predictor of homotopy class decision before collision avoidance has started, presented in [12], can be used as below

$$
\hat{\Theta}(\dot{\alpha})= \begin{cases}+\pi \text { or }-\pi, & \text { if } \dot{\alpha}=0 \\ +\pi, & \text { if } \dot{\alpha}>0 \\ -\pi, & \text { if } \dot{\alpha}<0\end{cases}
$$

where $\dot{\alpha}$ represents a derivative of the bearing angle. The boundary between homotopy class decisions ${ }^{1}$ in this case is the sign of $\dot{\alpha}$, denoted as $\operatorname{sign}(\dot{\alpha})$. In a noise-free observation of $\operatorname{sign}(\dot{\alpha})$ homotopy class decision ambiguity could only occur at the infinitesimal point $\dot{\alpha}=0$, however, due to noise in sensor readings this no longer holds.

\section{MODEL FOR OBSERVATION ERRORS FROM SENSOR}

Effective collaboration requires that every pairwise combination of crossing agents with collision risk choose the same homotopy class decision when taking their collision avoidance action. In the majority of cases this is trivial as $\operatorname{sign}(\dot{\alpha})$ can be perceived unambiguously as the same by both agents. However, whenever these perceptions differ the risk of ineffective collaboration rises as a consequence.

In this section a condition for ineffective collaboration and its risk is established based on a statistical properties of the noise in observation of the derivative of the bearing angle.

\section{A. Approximating the derivative of the bearing angle}

Let $\dot{\alpha}_{r, i}^{\text {real }}$ denote the noise-free derivative of the bearing angle between robots $r$ and $i$. For a robot to approximate its value consider a set $\vec{\alpha}=\left\{\alpha_{1}, \alpha_{2}, \cdots, \alpha_{T}\right\}$ of the $T$ last measurements of the bearing angle $\alpha$ done at fixed time intervals. Based on these samples of the bearing angle, its time derivative can be approximated with the symmetric

\footnotetext{
${ }^{1}$ The term "boundary between homotopy class decision" is sometimes shortened to just "boundary" within this work.
} 


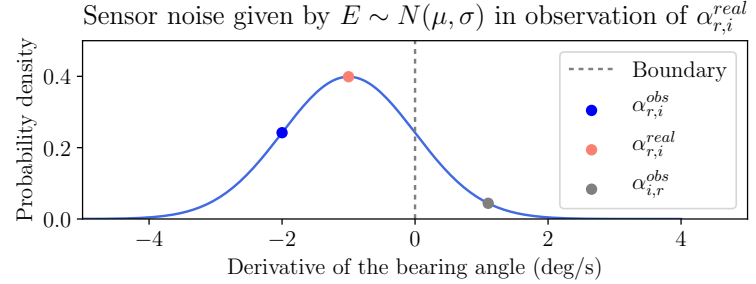

Fig. 3: Robots $r$ and $i$ observe different signs for $\dot{\alpha}_{r, i}^{\text {real }}$. Boundary refers to choice of distinct homotopy classes.

difference quotient as $\dot{\alpha} \approx\left[\alpha_{T}-\alpha_{1}\right] /[\Delta t]$ where $\Delta t$ is the time elapsed between $\alpha_{1}$ and $\alpha_{T}$ samples.

The value of $T$ balances a diverse set of trade-offs, for instance, a larger $T$ would decrease the impact of noise from individual samples, slow reaction times after each collision avoidance action due to additional bearing measurements and increase difference between the instantaneous value of $\dot{\alpha}$ and its approximation. In this work $T=5$ giving a minimum delay of $125 \mathrm{msec}$ between velocity changes in a PR2 robot (25 msec per scan).

Given this approximation of $\dot{\alpha}$, whenever $r$ observes the change in the bearing angle over time with respect to a robot $i$ it perceives this derivative value as $\dot{\alpha}_{r, i}^{\text {obs }}=\dot{\alpha}_{r, i}^{\text {real }}+\epsilon$ where $\epsilon$ denotes the error during observation of $\dot{\alpha}_{r, i}^{\text {real }}$. The robot $i$ will apply a similar approach and observe a likely distinct $\dot{\alpha}_{i, r}^{\text {obs }}$. As such, whenever $\dot{\alpha}_{r, i}^{\text {real }} \approx 0$ the fluctuations in observed values might lead to $\operatorname{sign}\left(\dot{\alpha}_{i, r}^{\text {obs }}\right) \neq \operatorname{sign}\left(\dot{\alpha}_{r, i}^{\text {obs }}\right)$. In such case, robots would incorrectly attempt to cross each other on opposite sides during avoidance.

\section{B. Ineffective collaboration risk with model of sensor error}

Evaluating the chance of ineffective collaboration requires first understanding the error distribution for observations of the derivative of the bearing angle. From this, a probability $P\left(\operatorname{sign}\left(\dot{\alpha}_{i, r}^{\mathrm{obs}}\right) \neq \operatorname{sign}\left(\dot{\alpha}_{r, i}^{\mathrm{obs}}\right)\right)$ can be calculated so that our mitigation of ineffective collaboration can be presented.

From the perspective of robot $r$ and without communication with a robot $i$ the values of $\dot{\alpha}_{r, i}^{\text {real }}$ or $\dot{\alpha}_{i, r}^{\text {obs }}$ are unknown. Consider that $r$ samples the observations of $\dot{\alpha}_{r, i}^{\text {real from a }}$ normally distributed random variable $\mathrm{A} \sim \mathcal{N}(\mu, \sigma)$ where the unknown mean is $\mu=\dot{\alpha}_{r, i}^{\text {real }}$ and the variance is $\sigma$. The variance is calculated at each time-step based on the sensor type and situational factors of $r$ and $i$ as $\sigma_{r, i}$ which are detailed in Sec. VII.

The risk of ineffective collaboration is associated with the boundary between homotopy class decisions. Even with unknown values, properties of the average distance between samples of a normal distribution can approximate the chance of mismatch between $\operatorname{sign}\left(\dot{\alpha}_{r, i}^{\text {obs }}\right)$ and $\operatorname{sign}\left(\dot{\alpha}_{i, r}^{\text {obs }}\right)$. To that end, our first objective is to determine the probability that the distance between $\dot{\alpha}_{r, i}^{\text {obs }}$ and the unknown $\dot{\alpha}_{i, r}^{\text {obs }}$ is large enough to cross the homotopy class decision boundary. The fact that the perceived distance to the boundary can change, as seen in Fig. 3, does not affect the statistical properties of the result as it is solely used as a risk indicator.
The probability density function (pdf) of the distance between elements sampled from a standard normal distribution was presented in [14]. In our work this derivation was done while assuming a non-standard normal distribution, yielding

$$
f_{\sigma}(x)=\frac{\exp \left(\frac{-x^{2}}{4 \sigma^{2}}\right)}{\sigma \sqrt{\pi}}
$$

Although the value of $\dot{\alpha}_{r, i}^{\text {real }}$ is unknown, assessing whether there is risk of ineffective collaboration can still be calculated. First by integrating the perceived distance pdf

$$
g_{\sigma}(\dot{\alpha})=\int_{|\dot{\alpha}|}^{\infty} f_{\sigma}(x) d x
$$

and second by considering that crossing the boundary happens only in a single direction, as can be seen in Fig. 3. As such, the associated risk of ineffective collaboration should be halved. Finally, the predicate to determine whether the risk of ineffective collaboration is sufficient to entail our mitigation strategy is then given by

$$
[1 / 2] \cdot g_{\sigma}(\dot{\alpha}) \geq 1-\Lambda
$$

where in this work $\Lambda=90 \%$ so there is only $1 \%$ chance of ineffective collaboration after two consecutive decisions.

This risk assessment strategy allows our strategy for mitigation of ineffective collaboration to be finally presented.

\section{Mitigation of InefFective COLlaboration}

In a near symmetry scenario a decision has to be made on how to deal with the nearing collision under the assumption that ineffective collaboration is possible. This section introduces measures that can be taken during this avoidance motion so that in the next decision $\operatorname{sign}\left(\dot{\alpha}_{r, i}^{\text {real }}\right)$ is more likely to be unambiguously perceived as the same by both agents.

\section{A. Velocity change impact on derivative of the bearing angle}

Whenever robot $r$ evaluates that collision avoidance with another robot $i$ is necessary, it changes its current velocity $\vec{v}_{r}$ to $\vec{v}_{r}^{\text {new }}$ based on the velocity of $i$ denoted as $\vec{v}_{i}$. The robot $i$ will apply a similar logic to calculate $\vec{v}_{i}^{\text {new }}$.

These velocity changes, when observed separately, cause a change $\Delta \dot{\alpha}_{r, i}$ and $\Delta \dot{\alpha}_{i, r}$ in their observed instantaneous value (see Sec. 2.2.1 of [15] for equation). Its new instantaneous value will be approximately the sum of $\dot{\alpha}_{r, i}^{\text {real }}$ with these individual changes in the derivative of the bearing angle, given by

$$
\dot{\alpha}_{r, i}^{\text {new }}=\dot{\alpha}_{r, i}^{\text {real }}+\left(\Delta \dot{\alpha}_{i, r}+\Delta \dot{\alpha}_{r, i}\right)
$$

As can be seen in Fig. 4, the range of values $\Delta \dot{\alpha}_{r, i}$ can assume depending on the chosen velocity is significant. Thus, in case both $r$ and $i$ were to respectively sample the value of $\Delta \dot{\alpha}_{r, i}$ and $\Delta \dot{\alpha}_{i, r}$ from an uniform distribution with an appropriately chosen size, a confidence of $\Lambda$ could be obtained that their sum is larger than a certain threshold. As a consequence, the next perceived value of $\dot{\alpha}_{r, i}^{\text {real }}$ would be far enough from the boundary to allow for unambiguous crossing order observation. 


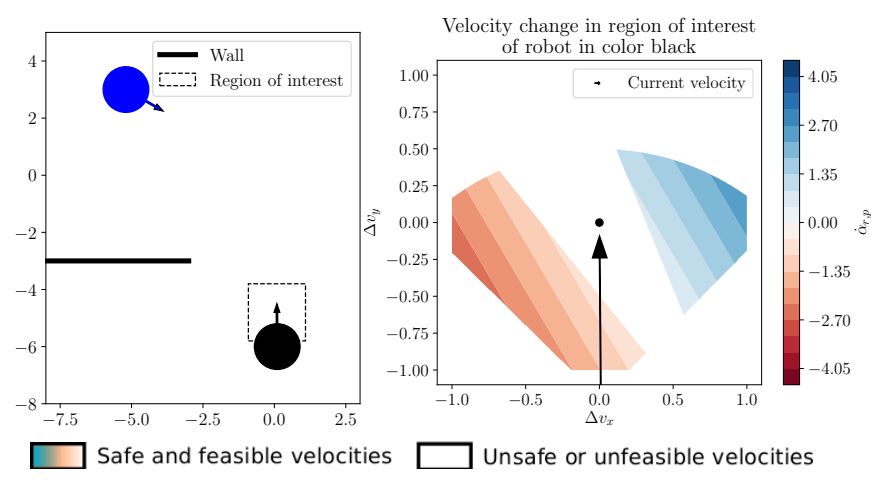

Fig. 4: Velocity change effect in the derivative of the bearing angle. Colorbar is discretized to facilitate visualization.

\section{B. Bounds length for near symmetry mitigation}

The framework on Sec. V-A can utilize statistical properties of uniform distributions to obtain a confidence in the distance between the values of $\Delta \dot{\alpha}_{i, r}$ and $\Delta \dot{\alpha}_{r, i}$.

To that end, the first step is to find the mitigation probability $P(\mathcal{M})$ required to reach $\Lambda$ confidence through a joint product between two independent events: worst case chance of robots misjudging crossing order $(50 \%)$ and chance of failing to mitigate symmetry. Isolating $\mathcal{M}$ yields

$$
P(\mathcal{M})=1-\frac{(1-\Lambda)}{[1 / 2]}=2 \Lambda-1
$$

Let $k$ denote the desired minimum distance between samples in both directions, unlike in (4) where only a single direction was used, its value can be calculated as

$$
k=g_{\sigma}^{-1}(1-P(\mathcal{M}))
$$

Our intention is for each robot to sample from an uniform distribution $\mathcal{X}(0, L)$ in order ensure that the equality $P\left(\left|X_{r}-X_{i}\right|>k\right)=\Lambda$ holds where $X_{r}$ and $X_{i}$ are independent samples by $r$ and $i$. The equation that relates $\Lambda$, the length $L$ of the uniform distribution and $k$ is demonstrated in Appendix C.1.2 of [15] and defined as

$$
\Lambda=(L-k)^{2} / L^{2} \text {, }
$$

Isolating $L$ yields $L^{2}(\Lambda-1)+2 L k-k^{2}=0$ which has two roots $L_{1}$ and $L_{2}$ from which the largest one is selected.

In the following section, the feasible upper and lower bounds for possible values of the derivative of the bearing angle due to velocity change are determined and actions in case it is larger or smaller than $L$ presented.

\section{AVOIDANCE MOTION UNDER NEAR-SYMMETRY}

This section describes a method to select an homotopy class that best respects the desired confidence in near symmetry mitigation while finding an avoidance velocity.

To generate collision avoidance velocities and calculate feasible bounds for the derivative of the bearing angle under several constraints a two-step optimization is used. First, random perturbations on the input are done which are followed by local optimizations using Sequential Least SQuares Programming (SLSQP) [16] as it can handle any combination of bounds, equality and inequality constraints.
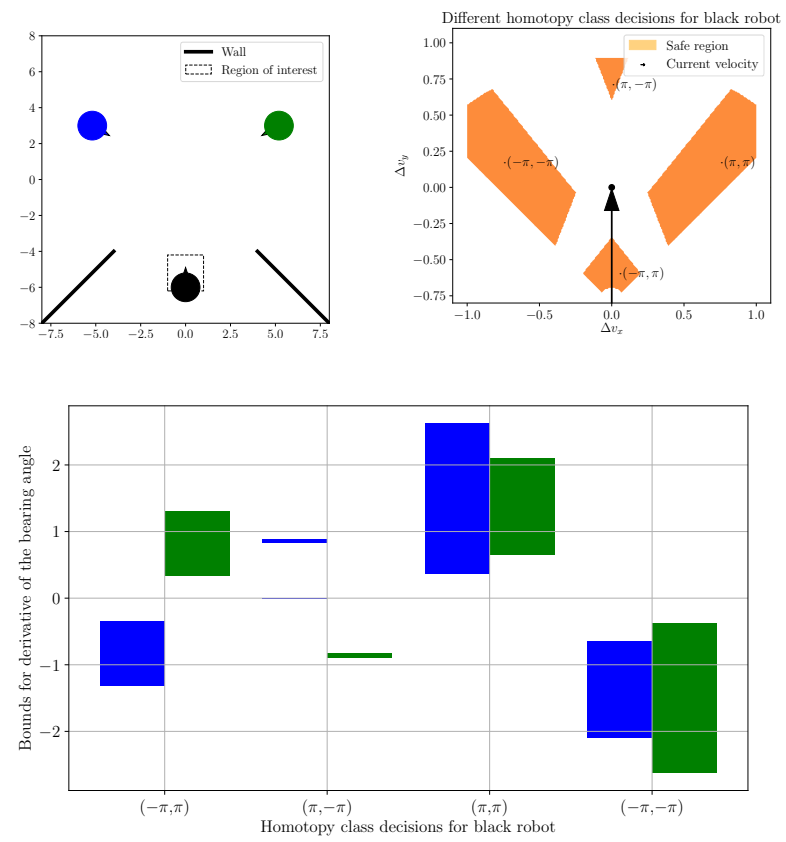

Fig. 5: Three-way situation with ambiguous crossing order. From the perspective of the black robot four homotopy class decisions are possible. Cross in front of others would not provide sufficient bounds for near symmetry mitigation.

\section{A. Enumerating homotopy classes to evaluate}

Mitigation of near symmetry requires an available range of velocity changes that may not be possible for a particular homotopy class decision, e.g. due to the presence of other obstacles. In these scenarios, a robot should explore other crossing sides in order to properly mitigate near symmetry. Thus, let $c \in\{-\pi, \pi\}$ denote an homotopy class decision for a robot with respect to another. The risk of ineffective collaboration for a given $c$ is

$$
h_{\sigma}(\dot{\alpha}, c)= \begin{cases}\frac{1}{2} \cdot g_{\sigma}(\dot{\alpha}), & \text { if } \hat{\Theta}(\dot{\alpha})=c \\ 1-\frac{1}{2} \cdot g_{\sigma}(\dot{\alpha}), & \text { otherwise }\end{cases}
$$

A robot $r$ observing a set of robots $i \in \mathcal{I}$ should ideally account for all possible homotopy class decisions. As this is impractical for larger number of robots, a subset is obtained by skipping the evaluation of unambiguous crossing side decisions using the predicate: $h_{\sigma}\left(\dot{\alpha}_{r, i}^{\mathrm{obs}}, c\right) \geq 1-\Lambda$.

\section{B. Feasible and safe bounds for near symmetry mitigation}

In Sec. $\mathrm{V}$, a method to calculate the necessary range of derivatives of the bearing angle in order to guarantee a certain confidence in near symmetry mitigation was introduced. In practice, the robot motion model and the configuration of obstacles in the environment impose a practical bound on the range of velocities and as consequence the range of derivative of the bearing angle that can be sampled.

As seen in Fig. 5, each homotopy class decision for $r$ allows for a distinct subset of velocities and also brings different bounds on values for the derivative of the bearing angle that still avoid future collision. These bounds are 
calculated by finding their minimum and maximum values while still constraining their signs to match the ones from the homotopy class decision and also account for safe velocities with respect to all other obstacles.

Let $B_{r, i}^{+}(\psi)$ and $B_{r, i}^{-}(\psi)$ denote for $r$ with respect to $i$ respectively the upper and lower bound of values for the derivative of the bearing angle for an homotopy class $\psi$. For each achievable homotopy class, in decreasing order of confidence in effective collaboration, the first where $L_{r, i} \leq$ $B_{r, i}^{+}(\psi)-B_{r, i}^{-}(\psi)$ for all $i \in I$ is chosen. In case no matching homotopy class is found the closest one to fulfilling the condition is selected. Finally, given this homotopy class, an avoidance motion can be calculated in the following section.

\section{Avoidance velocity with near symmetry mitigation}

A collision avoidance for near symmetry scenarios intends to avoid collision while guaranteeing a certain confidence in effective collaboration in case of ambiguous crossing order. Thus, our termination condition is that the roles of each robot are unambiguous for a homotopy class decision, in which case any standard collaborative approach would suffice.

Each agent will minimize the change in their desired velocity $\vec{v}_{r}^{\text {des }}(t)$ to a value that avoids future collision using

$$
\vec{v}_{r}^{*}=\underset{\vec{v} \in \mathcal{F}_{r}^{p}}{\arg \min }\left\|\vec{v}-\vec{v}_{r}^{\mathrm{des}}(t)\right\|
$$

where $\mathcal{F}_{r}^{p}$ is the set of velocities for $r$ where the closest predicted future distance between the circumscribed radii of $r$ and all $i \in \mathcal{I}$ is larger than the threshold for collision risk.

The mitigation approach presented requires respecting the chosen homotopy class and also a uniform sample of a derivative of the bearing angle value from a distribution of length $L_{r, i}$ from $r$ with respect to each agent $i$. To solve both of these, let $u_{r, i}=R\left(0, L_{r, i}\right)$ represent a random value sampled within the interval $\left[0, L_{r, i}\right]$. This value is sampled once for each $i \in \mathcal{I}$ prior to the optimization to fulfill both aforementioned requirements within the constraint

$$
\dot{\alpha}_{r, i}^{\text {new }}=B_{r, i}^{-}(\psi)+u_{r, i}, \forall i \in \mathcal{I}
$$

in practice, due to the difficulty of specifying exact values for more than one distinct derivative of the bearing angle, in case no optimization solution is found, the equality constraint for the robot with the least risk of ineffective collaboration is removed and the optimization is restarted.

As robots can continuously fail to establish unambiguous crossing order, despite mitigation, a speed limit based on distance is added to avoid accidental collision.

\section{Simulated EXPERIMENTS}

This section intends to showcase robot specific calibration for sensor noise and test limits of the presented approach during mitigation. First a comparative validation is done of the presented approach against a standard navigation method in symmetric scenarios. Afterwards, experiments in a more realistic simulation are presented using Gazebo-simulated Personal Robot 2 (PR2) [17] robots controlled using the Robot Operating System (ROS).

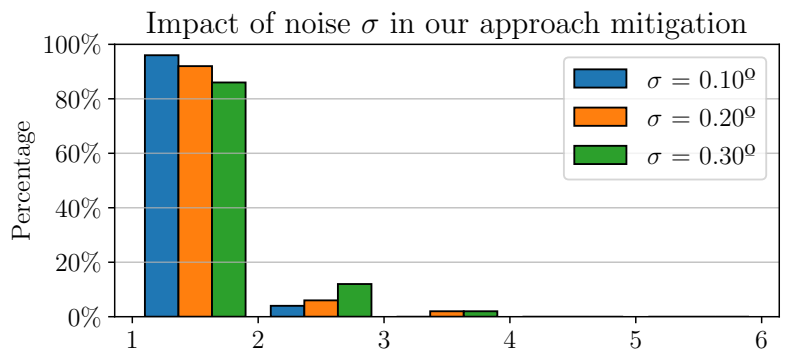

Fig. 6: Noise impact mitigation performance for two robots.

Experiments were done considering the worst case scenario, that is, in fully symmetric situations with $\dot{\alpha}=0$ for all pairwise combinations of robots.

\section{A. Large-scale comparative analysis of mitigation impact}

The presented approach is compared to Reciprocal Velocity Obstacles (RVO) [6], [7], a standard multi-agent navigation method. As RVO agents have perfect sensing, a modified version of RVO was generated in which noise was added to the observations of each robot to all others robots in the environment. The noise was normally distributed following the behavior described in Sec. VII-B.

This experiment is done by measuring the number of decisions until the chance of effective collaboration is above the desired confidence. All tests were repeated 200 times and results are shown in Fig. 7 and examples in Fig. 8. With two robots mitigation allows for unambiguous crossing order on at most two decisions $(250 \mathrm{~ms})$ in upwards of $99 \%$ of the tests while RVO with noise required up to 5 decisions $(625 \mathrm{~ms})$. Mitigation between three robots more than doubles the chance of disambiguation after one decision.

Mitigation performance is likely to surpass $\Lambda$ when $L$ is smaller than the bounds for the derivative of the bearing angle due to the fact that its next measurement is not made instantaneously and a non-zero value will increase as robots approach their crossing point (see examples in Fig. 2).

Finally, the impact of distinct noise levels in mitigation efficiency was evaluated as shown in Fig. 6 with positive results. The noise values $\sigma=0.1^{\circ}, \sigma=0.2^{\circ}$ and $\sigma=0.3^{\circ}$ allowed testing low, medium and high levels of noise.

\section{B. ROS-integrated experiments}

This experiment replicates scenarios where 2 or 3 fullysimulated PR2 robots attempted to avoid collision in near symmetry situations. A comparison of our approach with RVO with noise in ROS is available in video ${ }^{2}$.

As a robot $r$ model requires a $\sigma_{r, i}$ error distribution to predict average distance between samples of the derivative of the bearing angle (see Sec. IV-B). Near collision situations involving two PR2 robots, as shown in Fig. 9, were executed several times and the observed values of the derivative of the bearing angle until the crossing point were compared with their real values which were obtained directly from the simulator. The difference between observed value and

\footnotetext{
${ }^{2}$ See for experiments https://youtu.be/w9m5Tjj8eim
} 


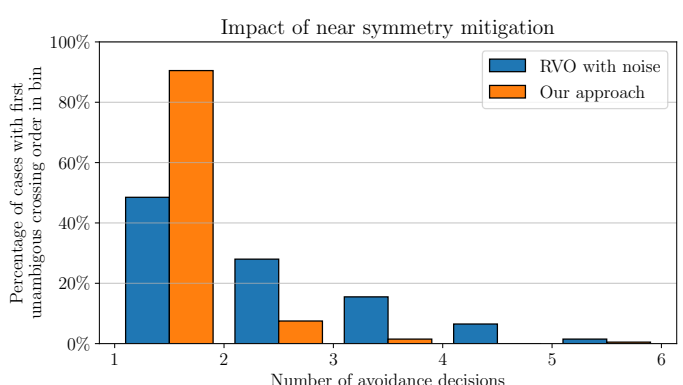

(a) A robot avoiding another with $\sigma=0.2$.

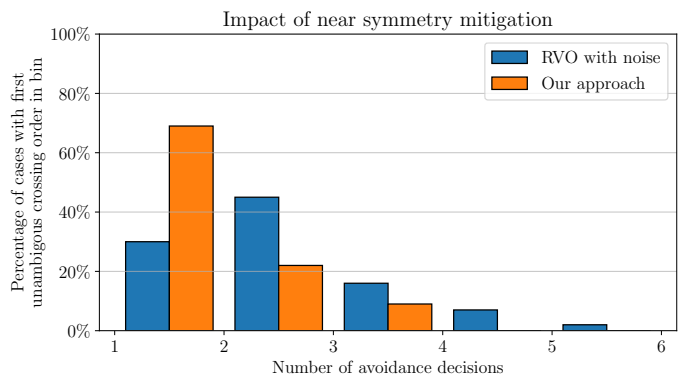

(b) A robot avoiding two others with $\sigma=0.2$

Fig. 7: Relating number of consecutive avoidance decisions with percentage of tests that had unambiguous crossing order.
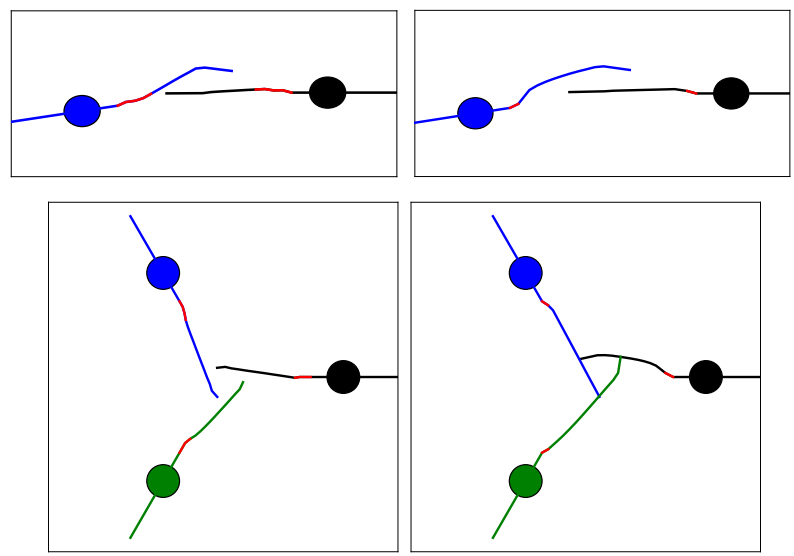

Fig. 8: Examples of difference in generated trajectories between methods. Left plots are RVO with noise and right plots are our approach. Red lines represent ineffective action(s).
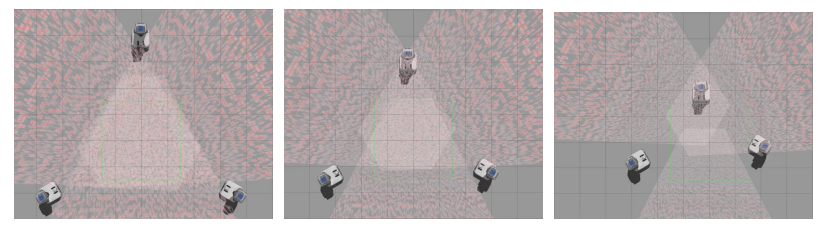

Fig. 9: Frames where three robots initially misjudge crossing order but our approach disambiguate it after one decision.

baseline was fitted to a Gaussian distribution and its standard deviation applied into (2). Thus, for situations with robots within less than 1 meter distance from each other $\sigma_{r, i}=1.17^{\circ}$ while within 2-4 meter distance an average of $\sigma_{r, i}=0.26^{\circ}$ was obtained. Although exploiting holonomic motion properties by avoiding changing the yaw of the PR2 during avoidance motion decreased sensor noise, further improvement seems possible.

\section{DISCUSSION}

Mitigation of ineffective collaboration in near symmetric scenarios was done through exploitation of properties of normal and uniform distributions. The results showed near optimal mitigation in simulated scenarios with a model that can be directly replicated and verified. Commonly considered alternatives to this problem have major limitations, as

1) Always crossing in either left or right side could solve the problem, however, it would be less efficient in $50 \%$ of the cases due to role reversal. Some scenarios would be harder to solve due to obstacles.

2) Moving homotopy class boundary to non-zero value would be sensitive to noise in estimation of the instantaneous value for the derivative of the bearing angle.

For future work, it would be interesting to also evaluate actuation intensity for different near symmetry approaches.

\section{REFERENCES}

[1] P. Trautman and A. Krause, "Unfreezing the robot: Navigation in dense, interacting crowds," in Int. Conf. on Intelligent Robots and Systems. IEEE, 2010.

[2] J. Cutting, P. Vishton, and P. Braren, "How we avoid collisions with stationary and moving obstacles," American Psychological Association, vol. 102, no. 4, 1995.

[3] J. Ondrej, J. Pettré, A.-H. Olivier, and S. Donikian, "A synthetic-vision based steering approach for crowd simulation," ACM Trans. Graph., vol. 29, no. 4, 2010 .

[4] S. Quinlan and O. Khatib, "Elastic bands: Connecting path planning and control," in Int. Conf. on Robotics and Automation. IEEE, 1993.

[5] N. Bohórquez and P. Wieber, "Adaptive step rotation in biped walking," in Int. Conf. on Intelligent Robots and Systems. IEEE, 2018.

[6] J. Van den Berg, M. Lin, and D. Manocha, "Reciprocal velocity obstacles for real-time multi-agent navigation," in IEEE Int. Conf. on Robotics and Automation. IEEE, 2008.

[7] J. van den Berg, S. Guy, M. Lin, and D. Manocha, Reciprocal n-Body Collision Avoidance. Springer, 2011.

[8] D. Helbing and P. Molnár, "Social force model for pedestrian dynamics," Physical Review E, vol. 51, no. 5, 1995.

[9] F. Zanlungo, T. Ikeda, and T. Kanda, "Social force model with explicit collision prediction," EPL (Europhysics Letters), 2011.

[10] T. Dutra, R. Marques, J. Cavalcante-Neto, C. Vidal, and J. Pettré, "Gradient-based steering for vision-based crowd simulation algorithms," Computer graphics forum, vol. 36, no. 2, pp. 337-348, 2017.

[11] M. Kuderer, H. Kretzschmar, and W. Burgard, "Teaching mobile robots to cooperatively navigate in populated environments," in Int. Conf. in Intelligent Robots and Systems (IROS). IEEE, 2013.

[12] G. Silva, A.-H. Olivier, A. Cretual, J. Pettre, and T. Fraichard, "Effective Human-Robot Collaboration in near symmetry collision scenarios," in Int. Conf. on Robot and Human Interactive Communication (RO-MAN). New Dehli, India: IEEE, 2019.

[13] G. Alenyà, A. Négre, and J. L. Crowley, "A comparison of three methods for measure of time to contact," in IEEE/RSJ Int. Conf. on Intelligent Robots and Systems (IRg28OS), 2009, pp. 4565-4570.

[14] B. Thirey and R. Hickman, "Distribution of euclidean distances between randomly distributed gaussian points in n-space," arXiv arXiv:1508.02238, 2015.

[15] G. Silva, "Towards natural human-robot collaboration during collision avoidance,” Theses, Université Grenoble-Alpes, ED MSTII, Feb. 2020. [Online]. Available: https://tel.archives-ouvertes.fr/tel-02501417

[16] D. Kraft, "Tomp-fortran modules for optimal control calculations," Int. Trans. Math. Softw., vol. 20, no. 3, pp. 262-281, 1994.

[17] PR2, "Ieee pr2 website," https://robots.ieee.org/robots/pr2/, 2019, accessed: 2020-07-20. 